\title{
Cytomegalovirus Disease in Renal Transplant Recipients: A Single-Center Experience
}

\author{
Dharmendra Bhadauria $\cdot$ R. K. Sharma \\ A. Kaul · Narayan Prasad A Amit Gupta • \\ Anurag Gupta $\cdot$ Aneesh Srivastava
}

Received: 3 March 2011/Accepted: 17 March 2012/Published online: 4 April 2012

(C) Association of Microbiologists of India 2012

\begin{abstract}
Cytomegalovirus (CMV) is the most common viral infection following kidney transplant, has been recognized as a major factor for graft loss and increased incidence of acute rejection. Different studies have reported a variable incidence of CMV disease with the use of Mycophenolate mofetil (MMF). We retrospectively analyzed our renal transplant recipients to review the results of CMV disease and to compare CMV disease in patient on Azathioprine and MMF for this purpose we retrospectively reviewed 521 live related kidney transplant recipients at our institute. $74(14.2 \%)$ live related allograft recipients developed CMV disease after a median interval of $7.18 \pm 4.35$ months from transplantation. The mean age was $36.15 \pm 10.7$ years. 63 of the patients were male. Malaise, fever and diarrhea were among most common symptoms. $20(27.02 \%)$ of the 74 recipients developed transaminitis, $13(17.2 \%)$ developed CMV gastritis, 5 $(9.13 \%)$ recipients developed pneumonia, and $3(4.05 \%)$ patient developed colitis. $59(80 \%)$ patients had leucopenia and $41(56.5 \%)$ developed thrombocytopenia. Mean serum creatinine level was $1.5 \pm 0.4(0.9-2.4) \mathrm{mg} / \mathrm{dl}$ before the disease, $1.9 \pm 0.6(1.3-3.6) \mathrm{mg} / \mathrm{dl}$ at the time of the diagnosis, and $1.7 \pm 0.06(0.8-4.2) \mathrm{mg} / \mathrm{dl}$ at the end of the treatment. CMV disease developed in $9(36 \%)$ of recipients who received basiliximab as induction therapy
\end{abstract}

D. Bhadauria $(\varangle) \cdot$ R. K. Sharma · A. Kaul · N. Prasad ·

A. Gupta · A. Gupta

Department of Nephrology, Sanjay Gandhi Post Graduate Institute of Medical Sciences, Raebareli Road, Lucknow, India e-mail: drdharm1@rediffmail.com

\section{A. Srivastava}

Department of Renal Transplantation, Sanjay Gandhi Post Graduate Institute of Medical Sciences, Raebareli Road, Lucknow, India and $13(30.24 \%)$ of recipients who received ATG $(p>0.05)$. The incidence of CMV disease was similar in cyclosporine based regimen (13.2\%) and Tacrolimus based regimen $27(16.16 \%)(p=0.137)$ and was also similar in Azathioprine $41(9.5 \%)$ and MMF group 33 (14.3\%) $(p=0.163)$. There was no significant difference in severity of CMV disease in both groups, except a higher incidence of leucopenia in Azathioprine group (86 vs. $74 \%, p<0.05$ ) as compared to MMF group. $51(68.91 \%)$ patient developed graft dysfunction during CMV disease. In conclusion we report a low incidence $(14.2 \%)$ and milder form of cytomegalovirus disease at our center. Use of universal cytomegalovirus prophylaxis was associated with a low incidence and milder form of the disease. Incidence of CMV disease was similar between Azathioprine and MMF groups.

Keywords Cytomegalovirus · CMV .

Renal allograft recipient

\section{Introduction}

Currently, cytomegalovirus (CMV) is a common cause of morbidity and mortality among patients receiving chronic maintenance immunosuppression [1] and is often considered the most important infection [2]. About one-quarter of renal transplant recipients developed CMV disease [3]. Generally, the highest incidence of CMV disease occurs during the first 3 months post-transplant [4], and is most severe in CMV naïve (CMV seronegative) recipients who receive an organ from a CMV seropositive donor ( $\mathrm{D}+/ \mathrm{R}-)$ [5-8]. Other risk factors for CMV disease include graft rejection and increased levels of immunosuppressive therapy, including anti-lymphocyte therapy, high dose 
corticosteroids, and Mycophenolate mofetil (MMF) [9-11]. Severe graft rejection and increased immunosuppression may cooperatively stimulate the development of active CMV infection in renal transplant recipients and CMV may activate the immune response to the graft [12].

CMV disease is an independent risk factor for acute rejection within the first 100 days and for recipient mortality [12]. In addition, the incidence of diabetes mellitus is higher in renal transplant recipients who developed CMV disease [5].

Increased incidence of CMV infection seen in patient on MMF [5-7, 11, 13]. Some studies have shown a slight increase in incidence and tissue invasive disease in patients being treated with MMF of 2 or $3 \mathrm{~g} /$ day compared with placebo or Azathioprine [2, 8-10, 12]. There is paucity of data regarding CMV disease in renal transplant recipients in Indian subcontinent. So we undertook this retrospective study to analyze the results of CMV disease in renal transplant recipients in Indian population and to compare CMV disease in patient on Azathioprine and MMF.

\section{Patients and Methods}

We retrospectively reviewed all regularly followed up, live related kidney transplant recipients, at our institute since January 2003-June 2009.

Five hundred and twenty-one renal transplant recipients (including children and adults) were studied who were transplanted from January 2003 to June 2009. The data was analyzed retrospectively using case notes, follow up records and using Hospital information system (a computer based programme), which is used for extracting validated investigational and patient related information. All patients and their donor who included had Ig M antibody negative and Ig G antibody positive status for CMV pretransplant.

We routinely follow a triple immunosuppression regimen including steroid, calcineurin inhibitors [(CNI) (either cyclosporine/Tacrolimus)] and Azathioprine or MMF as third drug. Use of IL-2 receptor blockers was optional based on patient's choice. This was given as Basiliximab $20 \mathrm{mg}$ on day 0 and day 4 or Daclizumab in 5 doses every fortnightly. Anti-thymocyte globulin (ATG) was used for induction in high risk patients and for treatment of acute rejection. In view of live donor renal transplants, delayed graft function was seen very infrequently. Either cyclosporine or Tacrolimus was used as CNI in a dose of $8 \mathrm{mg} /$ $\mathrm{kg}$ body weight or $0.2 \mathrm{mg} / \mathrm{kg}$ respectively. Cyclosporine dose was monitored according to $\mathrm{C}_{2}$ levels and dose was adjusted accordingly, keeping the $\mathrm{C}_{2}$ levels close to $800 \mathrm{ng} / \mathrm{ml}$ after 6 months of renal transplant. Steroids were started at $20 \mathrm{mg}$ on day 1 , and according to our protocol were tapered by $2.5 \mathrm{mg}$ per week, during the $3 \mathrm{rd}$ month post transplant and finally keeping the maintenance dose of $7.5-10 \mathrm{mg} /$ day through out the follow up.

All cases with clinical suspicion of rejection or rise in creatinine to a significant level underwent graft biopsy. This was considered after ruling out CNI nephrotoxicity, infections and other factors for acute graft dysfunction. However, in few circumstances like refusal by the patient or deranged parameters, treatment for presumed rejection was considered with pulse methyl prednisolone. Only biopsy proven severe vascular rejections were primarily treated with ATG or OKT3. Most of the patients were primarily treated with pulse methyl prednisolone, and anti-thymocyte globulin or OKT3 was used in event of steroid resistance. Since being a short follow up, acute rejection within 1 year of transplant were analyzed. Treatment for severe vascular rejection was given with $1.5 \mathrm{mg} / \mathrm{kg}$ of body weight of ATG on alternate day basis for 3-7 doses, depending on response or OKT3 at a dose of $5 \mathrm{mg}$ on alternate day for 3-7 doses. Patients with very severe acute vascular rejection not responding to anti-thymocyte globulin were given OKT3 or visa versa. Prophylaxis against CMV with 14 days of parenteral and then oral Ganciclovir was given for total of 12 week to patients who were treated with anti-thymocyte globulin or OKT3 for severe vascular rejection.

CMV disease was defined as the presence of fever for at least 3 days with one or more of the signs, either clinical or laboratory. Leucopenia $\left(<3,500\right.$ cells $\left./ \mathrm{mm}^{3}\right)$, thrombocytopenia $\left(<100,000\right.$ platelets $\left./ \mathrm{mm}^{3}\right)$, liver dysfunction (SGOT/ SGPT $>75 \mathrm{IU})$, Graft infection in form of intracytoplasmic inclusion bodies surrounded by inflammatory cells (on biopsy for acute graft dysfunction), retinitis, pneumonitis or gastrointestinal disease along with a positive CMV DNA by PCR. In few cases pp65 antigenemia was also available. All patients with confirmed CMV disease were treated with 2 weeks of intravenous Ganciclovir, followed by prophylaxis with oral Ganciclovir or Valaganciclovir for 3 months. During treatment with Ganciclovir, Azathioprine or MMF was decreased similarly in correspondence to TLC. Graft survival was calculated for 1 year for both groups independent of whether patient had CMV disease or not. Graft survival for 1 year was also calculated for patients who developed CMV disease form the onset of CMV disease.

\section{Statistics}

All variables were tested using the $t$ test or $\chi^{2}$ test to compare the demographic characteristics. Student $t$ test was used for statistical analysis. Kaplan-Meier survival analysis with log rank test was performed for survival of the grafts in patients who developed CMV disease on MMF or Azathioprine. A $p$ value of $<0.05$ was considered statistically significant. All statistical analysis was performed using SPSS 11.5 software package. 
Table 1 Demographic characteristics of renal allograft recipients

\begin{tabular}{ll}
\hline Variable & $N$ \\
\hline Total no of recipients & 521 \\
Recipient age (in years) & $36.15 \pm 10.7$ \\
Recipient gender (male) & $453(87 \%)$ \\
Cyclosporine based & $354(67.94 \%)$ \\
Tacrolimus based & $167(32.06 \%)$ \\
On AZA & $288(55.27 \%)$ \\
On MMF & $233(44.21 \%)$ \\
Received IL-2 RB & $25(4.79 \%)$ \\
Received ATG & $43(8.21 \%)$
\end{tabular}

AZA Azathioprine, MMF Mycophenolate mofetil, IL-2 RB IL-2 receptor blocker, $A T G$ anti-thymocyte globulin

\section{Results}

We analyzed 521 live related 1st renal transplants, which were performed from January 2003 to June 2009 in a span of $6 \frac{1}{2}$ years and followed till December 2008. The demographic characteristics of renal allograft recipients are summarized in Table 1. The most common immunosuppressive regimen was cyclosporine-based (CsA) 354 $(67.94 \%)$ remaining was on Tacrolimus based regimen. The number of patients who received Azathioprine was 288 and 233 patients received MMF apart form CNI and Steroids according to the protocol regimens of our institute. 25 and 43 patient received IL-2 Receptor Blocker (IL-2RB) and ATG respectively.

Demographic characteristics of patients, who developed CMV disease and its manifestations, are shown in Table 2. 74 live related allograft recipients $(14.2 \%)$ developed CMV disease after a median interval of $7.18 \pm 4.35$ months from transplantation. All of the donor and recipients undergoing living transplantation was $\mathrm{CMV} \operatorname{IgG}$ seropositive. The mean age was $36.15 \pm 10.7$ years. 63 of the patients were men and 11 were women. 9 patients, who received basiliximab while 13, who received ATG, developed CMV disease. CMV disease developed in 7 (28\%) of recipients who received basiliximab as induction therapy and $13(30.24 \%)$ of recipients who received ATG $(p>0.05)$. Two of 10 recipients who were treated with both basiliximab and ATG developed CMV disease. CMV disease occurred in $17.1 \%$ of patients who did not receive induction therapy. The incidence of CMV disease was similar in CSA based regimen $(13.2 \%)$ and Tacrolimus based regimen 27 (16.16\%), $p=0.137 .51(68.91 \%)$ patient developed graft dysfunction during CMV disease. Malaise was the most common symptom, which was seen in $60(80 \%)$ patients. Fever and diarrhea was seen in $33(44.5 \%)$ and 28 (38\%) patients, respectively. $20(27.02 \%)$ of the 74 recipients developed transaminitis, $13(17.2 \%)$ developed CMV gastritis, 5 $(9.13 \%)$ recipients developed pneumonia, and $3(4.05 \%)$
Table 2 Demographic characteristics and manifestations of patients who developed CMV disease $(n=74)$

\begin{tabular}{|c|c|}
\hline Variables & Mean $\pm \mathrm{SD}$ \\
\hline Age of the recipient (years) & $39.65 \pm 11.6$ \\
\hline Gender (male) & $63(85.3 \%)$ \\
\hline $\begin{array}{l}\text { CMV serostatus in living before tra } \leq 0.05 \text { plantation } \\
\text { (D/R) positive/positive }\end{array}$ & $74(100 \%)$ \\
\hline Time to infection (Post tra $<0.05$ plant) months & $7.18 \pm 4.35$ \\
\hline Cyclosporine based & $47(13.2 \%)$ \\
\hline Tacrolimus based & $27(16.16 \%)$ \\
\hline On AZA & $41(9.5 \%)$ \\
\hline On MMF & $33(14.3 \%)$ \\
\hline Received IL-2 RB & $7(28 \%)$ \\
\hline Received ATG & $13(30.23 \%)$ \\
\hline \multicolumn{2}{|l|}{ Manifestations } \\
\hline Malaise & $60(80 \%)$ \\
\hline Fever & $33(44.5 \%)$ \\
\hline Diarrhea & $28(38 \%)$ \\
\hline Myalgia & $15(20.7 \%)$ \\
\hline Abdominal symptoms & $26(34.5 \%)$ \\
\hline Nausea-vomiting & $13(17.2 \%$ \\
\hline Coughing & $10(14 \%)$ \\
\hline $\begin{array}{l}\text { G.I involvement (oral ulcers, esophagitis, } \\
\text { duodenitis, pancreatitis) }\end{array}$ & $25(33.78 \%)$ \\
\hline Leucopenia & $59(80 \%)$ \\
\hline Thrombocytopenia & $41(56.5 \%)$ \\
\hline Liver dysfunction & $20(27.02 \%)$ \\
\hline Retinitis & $2(2.70 \%)$ \\
\hline Graft involvement (biopsy) & $4(5.40 \%)$ \\
\hline Pneumonitis & $5(9.13 \%)$ \\
\hline Colitis & $3(4.05 \%)$ \\
\hline
\end{tabular}

$C M V$ cytomegalovirus, AZA Azathioprine, MMF Mycophenolate mofetil, $I L-2 R B$ IL-2 receptor blocker, $A T G$ anti-thymocyte globulin

patient developed colitis. 59 (80 \%) patients had leucopenia and 41 (56.5\% developed thrombocytopenia. Mean serum creatinine level was $1.5 \pm 0.4(0.9-2.4) \mathrm{mg} / \mathrm{dl}$ before the disease, $1.9 \pm 0.6(1.3-3.6) \mathrm{mg} / \mathrm{dl}$ at the time of the diagnosis, and $1.7 \pm 0.06(0.8-4.2) \mathrm{mg} / \mathrm{dl}$ at the end of the treatment.

We performed renal biopsy in $21(28.37 \%)$ patients. The biopsy revealed acute rejection in 15 (13.51\%) patients while $2(2.70 \%)$ patient had borderline rejection. Renal viral involvement was detected on 3 (4.05\%) renal biopsy specimen. There was no mortality due to CMV disease in our series (Table 3).

All infected recipients were treated with intravenous Ganciclovir $2.5-5.0 \mathrm{mg} / \mathrm{kg} \mathrm{q} 12 \mathrm{~h}$ according to creatinine clearance for 21 days and then oral ganciclovir or Valaganciclovir for total of 6 months duration. In 25 patients, immunosuppressive treatment was modified by decreasing 
Table 3 Outcome of patients who developed CMV disease

Variables

Graft dysfunction during CMV disease

$51(68.91 \%)$

(Creatinine increasing by $0.5 \mathrm{mg} \%$ from baseline)

Acute rejection during CMV infection (biopsy proven)

$17(22.97 \%)$

History of acute rejection episodes

$46(62.16 \%)$

$C M V$ cytomegalovirus

dose. CNI and MMF were the most commonly modified immunosuppressive drugs. In seven patients, relapsing CMV disease occurred. Three of them received basiliximab, while two recipients received ATG as induction therapy.

Comparison of CMV disease in patient on Azathioprine and MMF are shown in Table 4.

The incidence of CMV disease was similar in Azathioprine $(9.5 \%)$ and MMF group (14.3\%), $p=0.163$. CMV disease occurred at a significantly $(p<0.05)$ earlier time in MMF group (5.0 \pm 3.5 months, range 2-14 months) compared to Azathioprine group $(9.4 \pm 5.2$ months, range 3-20 months) in the post transplant period. When analyzed for severity of the CMV disease, only the incidence of leucopenia was observed to be significantly $(p<0.05)$ more in Azathioprine group compared to MMF group. There was no difference among the two groups in terms of thrombocytopenia, liver dysfunction, retinitis, gastrointestinal involvement, retinitis, pneumonitis or graft involvement (biopsy proven). The graft survival at 1 year was $90.5 \%$ and between Azathioprine (89\%) and MMF (92\%) group was not significantly different $(p=0.37)$, when the entire cohort of 521 patients was analyzed.

\section{Discussion}

In our study, the overall incidence of CMV disease was $14.2 \%$, which is low in comparison of the previous reports. The incidence of the disease after renal transplantation had been defined in previous studies. In a study among 451 renal transplant recipients, CMV disease occurred in $24 \%$ of patients during the first 100 days [1] and in study of Brenan et al. [13] the overall frequency of CMV infection after kidney transplant varies from 50 to $80 \%$ of patients, whereas CMV disease is observed in $20-60 \%$ of recipients. The universal prophylaxis strategy may be responsible for low incidence in our patients. The median time between the transplantation and the presentation of sign and symptoms was $7.18 \pm 4.35$ months in our study, which supports previous studies [6].

There are well-defined risk factors for CMV disease, such as CMV serostatus of the donor and recipient, the immunosuppressive regimen, and the type of transplanted organ. In our study, all of the donors and recipients undergoing living transplantation were CMV IgG seropositive. The other important risk factor, immunoprophylaxis with ATG or basiliximab, was studied in a couple of studies. In our study, CMV disease developed in 28 and $30.23 \%$ of recipients who received basiliximab and ATG as induction therapy, respectively. The difference was not statistically significant. Lebranchu et al. [7] found that there was significantly less CMV infection in the group treated with basiliximab. In the same study, CMV disease was seen in 6 and $12 \%$ of patients treated with basiliximab and ATG, respectively, but the difference was not statistically significant. In the study of Mourad et al. [8] $21.2 \%$ of patients treated with basiliximab and $41.5 \%$ of
Table 4 Comparison of CMV disease between Azathioprine and MMF

$C M V$ cytomegalovirus

\begin{tabular}{llll}
\hline Variables & Azathioprine & MMF & $p$ value \\
\hline CMV disease & $41(9.5 \%)$ & $33(14.3 \%)$ & \\
Age of the recipient (years) & $39.5 \pm 11.1$ & $39.8 \pm 12.1$ & $>0.05$ \\
& $(12-65)$ & $(22-64)$ & \\
Gender (male) & $19(86.4 \%)$ & $16(84.2 \%)$ & $>0.05$ \\
Time to infection (post transplant) & $9.36 \pm 5.2$ months & $5.0 \pm 3.5$ months & $<0.05$ \\
& $(3-20$ months) & $(2-14$ months $)$ & \\
Leucopenia & $19(86 \%)$ & $14(74 \%)$ & $<0.05$ \\
Thrombocytopenia & $12(55 \%)$ & $11(58 \%)$ & $>0.05$ \\
Liver dysfunction & $6(27 \%)$ & $5(26 \%)$ & $>0.05$ \\
Retinitis & $2(9.1 \%)$ & $1(5.3 \%)$ & $>0.05$ \\
G.I involvement (oral ulcers, esophagitis, & $9(41 \%)$ & $6(32 \%)$ & $>0.05$ \\
$\quad$ duodenitis, pancreatitis) & $1(4.5 \%)$ & $1(5.3 \%)$ & $>0.05$ \\
Graft involvement (biopsy) & $1(4.5 \%)$ & $2(10.5 \%)$ & $>0.05$ \\
Pneumonitis & & 1 patient died & \\
\end{tabular}


recipients treated with ATG developed CMV infection, the difference between the groups being statistically significant $(p=0.025)$.

In our study, malaise, fever, and diarrhea were the most common symptoms, seen respectively in 60,33 , and 28 of 71 patients. The incidence of symptoms seen in our patients is similar with the reports of previous authors. Despite mild course of disease in most infected recipients, organ involvements can coexist. Nemati et al. and PourReza-Gholi et al. [9, 10] reported fever, nausea, and vomiting as the most commonly seen symptoms. Farrugia and Schwab [11] identified fever, malaise, and myalgia as the most common symptoms. In the study of Abbott et al. [12], pneumonia was seen in $17.1 \%$, hepatitis in $4.2 \%$, and complications of transplanted organ in $6.6 \%$ of renal transplant recipients hospitalized for CMV disease. In the study of Durlik et al. [14], the incidence of hepatitis, gastroenteritis, and pneumonitis was 46, 27, and $9 \%$, respectively. In our study, the most commonly involved organs were also the stomach, lung, and colon. The incidence of organ involvements is variable among different studies. Thus, it is difficult to compare the results. Elevated serum creatinine level, leucopenia, and thrombocytopenia are important laboratory manifestations of CMV disease [6, 13]. Leucocytosis is as important as leucopenia in the diagnosis of the disease. In our study, the incidence of leucopenia and leucocytosis was 34.5 and $20.7 \%$, respectively. Pour-Reza-Gholi et al. [10] defined the most common laboratory findings as elevated serum creatinine level $(71 \%)$, thrombocytopenia (43\%), leucocytosis (22\%), and leucopenia (21\%). In that study, leucocytosis was as common as leucopenia. In addition, an increase in serum creatinine level was detected in 10 of 29 patients, corresponding to $34.4 \%$ of recipients. Patients were treated with intravenous Ganciclovir, $2.5-5 \mathrm{mg} / \mathrm{kg} \mathrm{q} 12 \mathrm{~h}$ according to creatinine clearance for 21 days. All patients were treated successfully. No mortality and graft loss due to CMV disease was seen during the course of the disease. This finding, which is similar to other studies, indicates to us that CMV disease has a mild course and can be treated successfully. This can be explained by early diagnosis and treatment of the disease. Despite the existence of welldefined risk factors, the disease can be seen in patients who show no risk factors. In this present study, CMV disease was seen in recipients who were treated with basiliximab as induction therapy, similar to those who were treated with ATG. On comparison, incidence of CMV disease was low and similar between Azathioprine group and MMF group in our cohort (9.5\% in Azathioprine group and $14.3 \%$ in MMF group, $p=0.16$ ), which might be due to that fact that all our patients were seromatched before transplant and we routinely used Ganciclovir prophylaxis in patients who were treated with ATG for induction or severe acute rejections. Our incidence of CMV disease was similar to study by Munoz MA et al. [6], who showed incidence of $8.4 \%$ in patients on MMF and $3.6 \%$ in patients not on MMF. Similarly, study by Basic-Jukic et al. [15] showed incidence of CMV in $20 \%$ of patients on MMF. Patients on MMF tend to develop CMV disease at a earlier stage post transplant compared to Azathioprine in our study which was $5.0 \pm 3.5$ vs. $9.4 \pm 5.2$ months, $p<0.05$ with a median of 4 months vs. 8 months). This difference was also noted by some [16], but not by others [8]. In terms of severity of CMV disease, we could not find any difference in Azathioprine or MMF use except in terms of leucopenia which was significantly more with the use of Azathioprine (86 vs. $74 \%, p<0.05$ ). Though, some studies $[6,17]$ have also shown higher, but not statistically significant percentage of leucopenia with Azathioprine use when compared to MMF (2 g/day) in patients who developed CMV disease in their cohort. Only higher dose of MMF ( $3 \mathrm{~g} /$ day) was associated with an increased incidence of leucopenia in patients who developed CMV disease when compared with Azathioprine [10, 12, 18].

Our study because of its retrospective nature has many limitations like ascertainment error, recall, informative censoring and lead-time biases, which were unavoidable. Our results should be confirmed by a larger prospective study with large number of patients.

Merits of our study are its large patient number and comparison of disease between MMF and Azathioprine group.

\section{Conclusion}

Thus, in the presence of nonspecific symptoms like fever, malaise, and diarrhea; as well as clinical manifestations such as leukopenia, leukocytosis, and elevated transaminase and creatinine levels, CMV disease should be suspected, even in low-risk patients. Incidence of disease is low and disease is mild, in high risk patients, on universal prophylaxis strategy. There is no difference among MMF and Azathioprine on incidence and outcome of CMV disease.

\section{References}

1. Murray BM, Brentjens J, Amsterdam D, Myers J, Gray V, Pawlowski I, Schewegler K, Singh JP (1997) Monitoring and diagnosis of cytomegalovirus infection in renal transplantation. J Am Soc Nephrol 8(9):1448-1457

2. Fishman JA, Rubin RH (1998) Infection in organ-transplant recipients. N Engl J Med 338(24):1741-1751

3. Pouteil-Noble C, Ecochard R, Landrivon G, Donia-Maged A, Tardy JC, Bosshard S (1993) Cytomegalovirus infection-an etiological factor for rejection? A prospective study in 242 renal transplant patients. Transplantation 55(4):851-857 
4. Schroeder R et al (2004) Cytomegalovirus disease latent and active infection rates during the first trimester after kidney transplantation. Transplant Proc 36(4):896-898

5. Murray BM, Subramaniam S (2004) Late cytomegalovirus infection after oral ganciclovir prophylaxis in renal transplant recipients. Transpl Infect Dis 6(1):3-9

6. Munoz MA, Andres A, Gallego R, Morales E, Morales JM, Aguado JM, Lumbreras C, Torres A, Rodicio JL, Praga M (2002) Mycophenolate mofetil immunosuppressive therapies increase the incidence of cytomegalovirus infection in renal transplantation. Transplant Proc 34:97

7. Lebranchu Y, Bridoux F, Büchler M, Meur Y, Etienne I, Toupance $O$ et al (2002) Immunoprophylaxis with basiliximab compared with antithymocyte globulin in renal transplant patients receiving MMF-containing triple therapy. Am J Trans 2:48-56

8. Mourad G, Rostaing L, Legendre C, Garrigue V, Thervet E, Durand D (2004) Sequential protocols using basiliximab versus antithymocyte globulins in renal-transplant patients receiving mycophenolate mofetil and steroids. Transplantation 78(4):584-590

9. Nemati E, Eizadi M, Moghani Lankarani M, Kardavani B, Khoddami-Vishteh H-R, Kalantar E et al (2007) Cytomegalovirus disease after kidney transplantation: clues to accurate diagnosis. Transplant Proc 39:987-989

10. Pour-Reza-Gholi F, Labibi A, Farrokhi F, Nafar M, Firouzan A, Einollahi B (2005) Signs and symptoms of cytomegalovirus disease in kidney transplant recipients. Transplant Proc 37:3056-3058

11. Farrugia E, Schwab TR (1992) Management and prevention of cytomegalovirus infection after renal transplantation. Mayo Clin Proc 67:879
12. Abbott KC, Hypolite IO, Viola R, Poropatich RK, Hshieh P, Cruess D et al (2002) Hospitalizations for cytomegalovirus disease after renal transplantation in the United States. Ann Epidemiol 6:402-409

13. Brennan DC (2001) Cytomegalovirus in renal transplantation. J Am Soc Nephrol 12(4):848-855

14. Durlik M, Siennicka J, Litwinska B, Majchrzak J, Trzcinska A, Samsel R et al (2001) Clinical manifestations and diagnosis of cytomegalovirus infection in renal allograft recipients. Transplant Proc 33:651-653

15. Basic-Jukic N, Kes P, Bubic-Filipi LJ, Puretic Z, Brunetta B, Pasini J (2005) Does Mycophenolate mofetil increase the incidence of cytomegalovirus disease compared with Azathioprine after cadaveric renal transplantation. Transplant Proc 37:850-851

16. Von Muller L et al (2006) Severe graft rejection, increased immunosuppression, and active CMV infection in renal transplantation. J Med Virol 78(3):394-399

17. Hagen M, Hjelmesaeth J, Jenssen T, Morkrid L, Hartmann A (2003) A six-year prospective study on new-onset diabetes mellitus, insulin release and insulin sensitivity in renal Transplant recipients. Nephrol Dial Transplant 18:2154-2159

18. Toda T, Motoki T, Kurosawa N, Owada E, Achiwa K, Yuhki Y, Tadano K, Takahashi Y, Shimoda N, Shindo J, Harada H, Seki T, Hirano T (2005) Relationship between dose of Mycophenolate mofetil and the occurrence of cytomegalovirus infection and diarrhea in renal transplant recipients. Yakugaku Zasshi 125(2): $177-185$ 